\title{
Reversible ageing of iridium oxide electrodes in acidic solutions*
}

\author{
H. ELZANOWSKA ${ }^{\ddagger}$, V. I. BIRSS ${ }^{\S}$ \\ Department of Chemistry, University of Calgary, Calgary, Alberta, Canada, T2N IN4
}

Received 21 July 1992; revised 11 November 1992

The cyclic voltammetric response of iridium oxide films, grown electrochemically in acidic solutions, can display a notable dependence on the time spent in the reduced state and on other factors such as the carrying out of very slow sweep rate experiments. This is seen primarily as a substantial positive shift of the anodic peaks and in a diminishment, by up to about $50 \%$, in the reaction kinetics. This effect, termed as film ageing, can be reversed, either by long times of cycling over an extended range of potential or by a brief holding period in the oxidized state of the film. Oxide films do not exhibit ageing in alkaline solutions. It is, therefore, suggested that film ageing is associated primarily with the loss of water from the film in acidic solutions at negative potentials, resulting in the inhibition of subsequent ion/solvent transport processes in the film.

\section{Introduction}

There has been a great deal of interest in the electrochemical behaviour of iridium oxide electrodes for a number of years [1-26]. This has stemmed both from its interesting electrochemistry and also due to its numerous potential practical applications, for example in display devices (due to its electrochromic properties $[4,5])$, as a neural stimulating electrode $[25,26]$, and also because of its potential as a cathode for advanced rechargeable batteries. For all of these purposes, it is desirable that the oxide film be chemically/physically stable and that it displays rapid and reproducible oxidation/reduction kinetics.

A detailed study by Mozota and Conway [7, 8] formed part of the pioneering investigations of electrochemically formed iridium oxide films. The main redox peaks, $A_{1} / C_{1}$, were attributed to the $\operatorname{Ir}(\mathrm{III}) /(\mathrm{IV})$ redox process and the prepeak, $A_{0}$, was linked to the presence of anions in the oxide film. Further studies by Burke et al. [10-12] and later by Pickup and Birss [14-18] showed that electrochemically grown Ir oxide films display a greater than $60 \mathrm{mV} \mathrm{pH}$ dependence over a wide range of $\mathrm{pH}$, consistent with earlier reports [1]. Based on the chemical analysis of the contents of the oxide film in the oxidized and reduced states, in both acidic (HX) and alkaline (MOH) solutions [16], and on in situ mass measurements [24], the following reactions have been shown to describe iridium oxide electrochemistry in aqueous solutions:

$$
\begin{aligned}
& \left(\mathrm{IrO}_{2} n \mathrm{H}_{2} \mathrm{O}\right)_{5}+5 \mathrm{e}^{--}+7 \mathrm{H}_{3} \mathrm{O}^{+}+2 \mathrm{X}^{-} \\
\Longleftrightarrow & {\left[\mathrm{Ir}_{5} \mathrm{O}_{3}(\mathrm{OH})_{7} 5 n \mathrm{H}_{2} \mathrm{O}\right]^{2+}\left(\mathrm{X}^{-}\right)_{2}+7 \mathrm{H}_{2} \mathrm{O} }
\end{aligned}
$$

$$
\begin{gathered}
\left(\mathrm{M}^{+}\right)_{3}\left[\mathrm{Ir}_{5} \mathrm{O}_{10}(\mathrm{OH})_{3} 7 \mathrm{H}_{2} \mathrm{O}\right]^{3-}+5 \mathrm{e}^{-}+7 \mathrm{H}_{2} \mathrm{O} \\
\Longleftrightarrow\left(\mathrm{M}^{+}\right)\left[\mathrm{Ir}_{5} \mathrm{O}_{6}(\mathrm{OH})_{4} 10 \mathrm{H}_{2} \mathrm{O}\right]^{-}+2 \mathrm{M}^{+}+7 \mathrm{OH}^{-}
\end{gathered}
$$

In the present work, we have focussed primarily on elucidating some of the factors which are responsible for the instability of the iridium oxide electrochemical response, when particular solutions, electrochemical growth conditions and potentials are employed. Similar to prior references $[6,10,18]$, this will be referred to as film ageing phenomena, which can be either reversible or irreversible in nature in the case of iridium oxide electrodes. Importantly, both forms of ageing significantly influence the kinetics of the principal redox reaction. In this paper, the conditions which provoke iridium oxide ageing will be examined and an effort will be made to explain it in terms of structural/compositional changes which occur within the oxide film in relation to Reactions 1 and 2 . It is of interest that similar results to those shown here have recently been reported for a number of polymer modified electrodes, e.g. polyaniline, Nafion, etc. [27-32]. Also, analogous effects have been reported for oxides formed electrochemically at amorphous alloy electrodes [33].

\section{Experimental details}

\subsection{Equipment, cells and chemicals}

An EG\&G PARC 173 potentiostat was used with a PARC 175 universal programmer and a PARC 179 digital coulometer. Voltammograms were

* This paper is dedicated to Professor Brian E. Conway on the occasion of his 65th birthday and in recognition of his outstanding contribution to electrochemistry.

$\ddagger$ Present address: Department of Chemistry and Biological Sciences, Oregon Graduate Institute, Beaverton, OR 97006-1999, USA (on leave from the University of Warsaw, Department of Chemistry, Warsaw, Poland).

${ }^{\S}$ Author to whom all correspondence should be addressed. 
recorded on a Hewlett-Packard $7044 \mathrm{~A}$ or a BBC SE $780 \mathrm{X}-\mathrm{Y}$ recorder, while current and charge transients were collected and displayed by an HP7090 digital plotter. All electrochemical experiments were carried out in conventional three-compartment glass cells, under argon at room temperature. All reagents were Fisher certified or Reagent ACS grade and were used as received, and all water was triply distilled.

\subsection{Electrodes}

Working electrodes were constructed from glassmounted iridium wire $(99.9 \%$, Johnson Matthey, $0.5 \mathrm{~mm}$ diam.). All electrode areas referred to in this paper are geometric areas (about $0.1 \mathrm{~cm}^{2}$ ). All potentials are quoted relative to the saturated sodium calomel electrode (SSCE, $+236 \mathrm{mV}$ vs SHE) which was separated from the working electrode by a Luggin probe. The counter electrode was a large area platinum gauze located in a separate compartment.

\subsection{Electrode pretreatment and oxide growth}

Before oxide growth, the electrode was generally cleaned electrochemically [14] by holding the potential at $2 \mathrm{~V}$ vs $\mathrm{SSCE}$ in $10 \% \mathrm{H}_{2} \mathrm{SO}_{4}$ for about $5 \mathrm{~min}$. Another rapid and efficient cleaning method, not used previously by us for iridium oxide electrodes, involved placing the electrode into the reducing part of a natural gas/air flame for several seconds. To grow an iridium oxide film, the potential was cycled $\left(1 \mathrm{~V} \mathrm{~s}^{-1}\right)$ or pulsed at $1 \mathrm{~s}$ intervals between 0.24 and $1.31 \mathrm{~V}$ in sulphuric and nitric acid solutions. Depend- ing on the desired thickness of the oxide film, the time of potential cycling/pulsing was varied from a few minutes up to about $1 \mathrm{~h}$.

\subsection{Determination of quantity of hydrous iridium oxide}

The amount of Ir oxide film formed was assessed by measuring the charge passed in a slow sweep CV experiment [14-18] between particular potential limits. Slow potential sweeps are essential in order to achieve equilibrium within the oxide film, so that all of the iridium sites have time to react. For thick oxide films, scan rates in the range of 1 to $20 \mathrm{mV} \mathrm{s}^{-1}$ were required for charge measurement, while in the case of thin oxides, higher sweep rates of up to $100 \mathrm{mV} \mathrm{s}^{-1}$ could be used. The positive potential limit $\left(E_{+}\right)$used for charge calculations was $1.0 \mathrm{~V} \mathrm{[15]} \mathrm{in}$ both $0.5 \mathrm{M} \mathrm{H}_{2} \mathrm{SO}_{4}$ and $1 \mathrm{M} \mathrm{HNO}_{3}$ solutions. The charges, which are considered to be proportional to the oxide film thickness, are represented by a charge enhancement factor (CEF) [7-9, 14, 15], considered to be a measure of the number of monolayers of iridium oxide film present. The CEF is equivalent to the ratio of the oxide charge density after a particular growth period to the charge in the first scan of potential at a fresh iridium electrode (about $0.11 \mathrm{mC} \mathrm{cm}^{-2}$ [15]). Iridium oxide films characterized by $\mathrm{CEF}$ values in the ranges of $40-80,80-280,280-500$ and beyond 500 are classified in this work as thin, medium, thick and very thick, respectively. Based on our previous work [15], a CEF of unity was shown by SEM studies to be equivalent to a dry film thickness of about $15 \AA$.

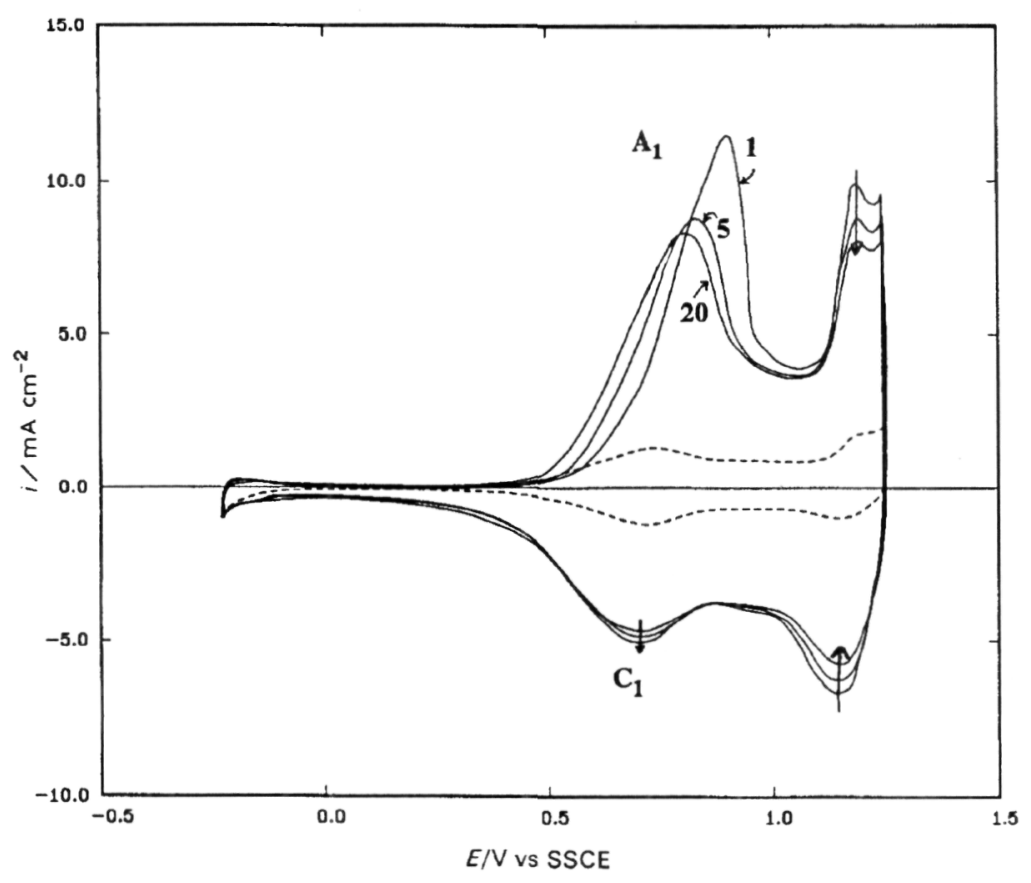

Fig. 1. Cyclic voltammetric response, $100 \mathrm{mV} \mathrm{s}^{-1}$, of thick ( $\mathrm{CEF}=282, \longrightarrow$ ) and thin $(\mathrm{CEF}=61,---)$ iridium oxide film in $0.5 \mathrm{M}$ $\mathrm{H}_{2} \mathrm{SO}_{4}$ with continuous cycling of potential after oxide growth and potential holding at $-0.24 \mathrm{~V}$ for 5 min. Cycle numbers after holding are shown on the $\mathrm{CVs}$. 

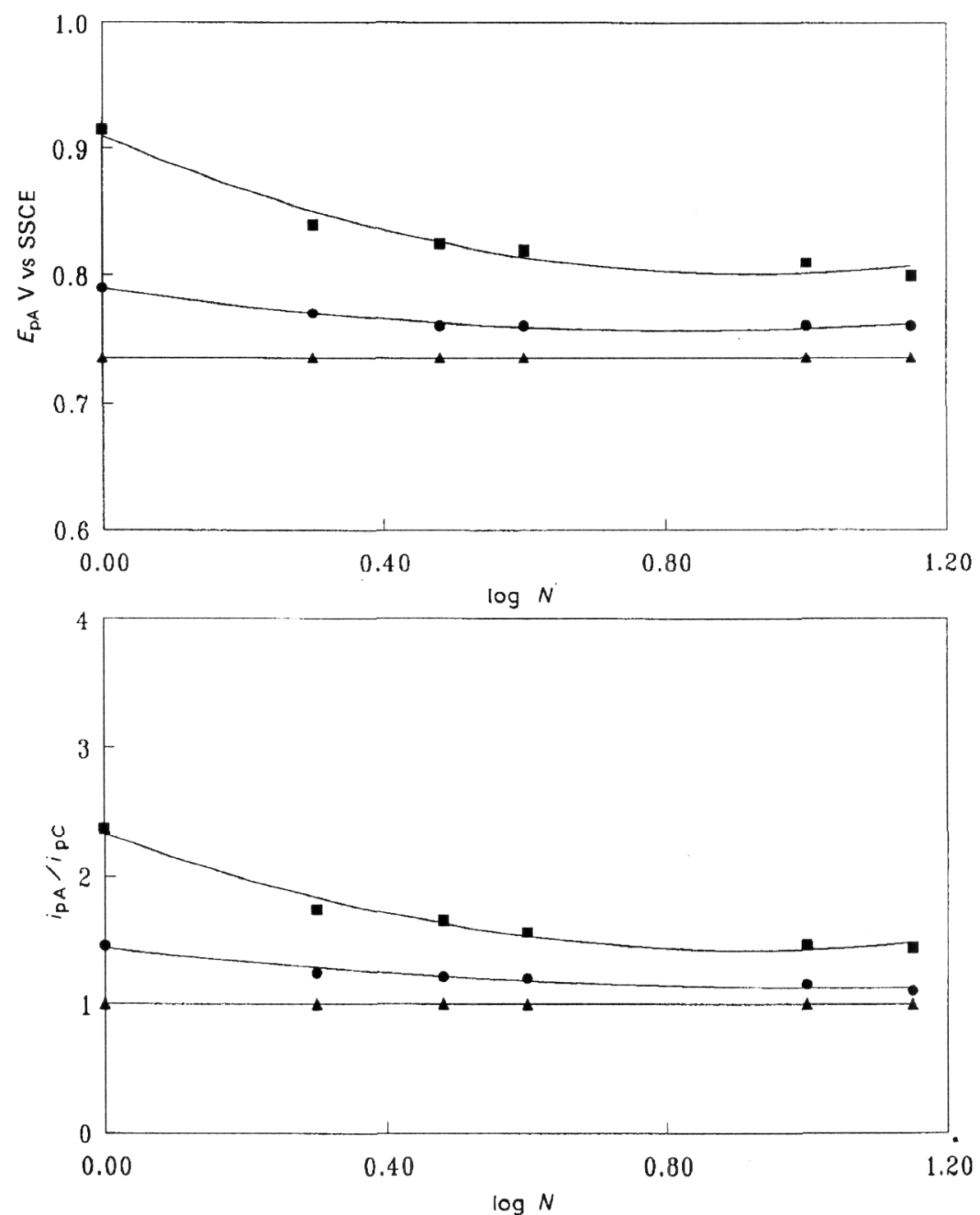

Fig. 2. (a) Peak potential of $\mathrm{A}_{1}$ against $\log N$, where $N$ is number of cycles applied after oxide growth and holding at $-0.24 \mathrm{~V}$ for $5 \mathrm{~min}$ in $0.5 \mathrm{M} \mathrm{H}_{2} \mathrm{SO}_{4}$. Oxides studied were thin $(\mathrm{CEF}=61, \boldsymbol{\Delta})$; medium thick $(\mathrm{CEF}=150, \bullet)$ and thick $(\mathrm{CEF}=282$, $\mathbf{m})$. (b) Ratio of currents of peak $\mathrm{A}_{1}$ to peak $\mathrm{C}_{1}$ against $\log N$, for same oxides as in Fig. 2(a).

\subsection{Electrode transfer experiments}

The transfer of iridium oxide electrodes between solutions was performed by first removing the electrode at a particular potential and dipping it several times in the new solution in a separate vessel for a few seconds, long enough to rinse the oxide surface but probably not sufficiently long to fully exchange the ionic contents of the film. The iridium oxide electrode was then transferred at the desired potential to a fresh aliquot of this solution in an electrochemical cell.

\section{Results and discussion}

3.1. General description of reversible ageing of iridium oxide

Figure 1 shows the cyclic voltammetric (CV) response of both a thin $(\mathrm{CEF}=61)$ and thick $(\mathrm{CEF}=282)$ freshly grown iridium oxide film in $0.5 \mathrm{M}$ sulphuric acid in about 20 potential scans immediately after oxide growth. The oxide films were grown by pulsing the potential between $E_{-}=-0.24$ and $E_{+}=1.2 \mathrm{~V}$, followed by holding at $E_{-}$for about $5 \mathrm{~min}$. It can clearly be seen that there is no change in the electrochemical response of the thin film in the first and subsequent cycles of potential. However, in the case of the thicker film, the electrochemical response changes significantly until a steady state CV is obtained. Moreover, if the potential is held again at $E_{-}$for $5 \mathrm{~min}$ after equilibration, a $\mathrm{CV}$ response similar to that seen in the first scan in Fig. 1 is obtained.

These changes in the CV signature, brought about particularly by time spent in the reduced state and as the oxide film thickness is increased, are referred to as ageing effects in this paper, primarily because these films display reduced kinetics (see later in this paper) as compared to unaged films, i.e. those which yield the equilibrated $\mathrm{CV}$ response shown in Fig. 1. The term 'ageing' has been used previously $[6,10$, 18 ] to describe iridium oxide films exhibiting a deteriorating response.

Figures 2(a) and (b) more clearly depict the changes of the $A_{1}$ peak potential and of the ratio of currents of peaks $A_{1}$ to $C_{1}$ for iridium oxide films of different 


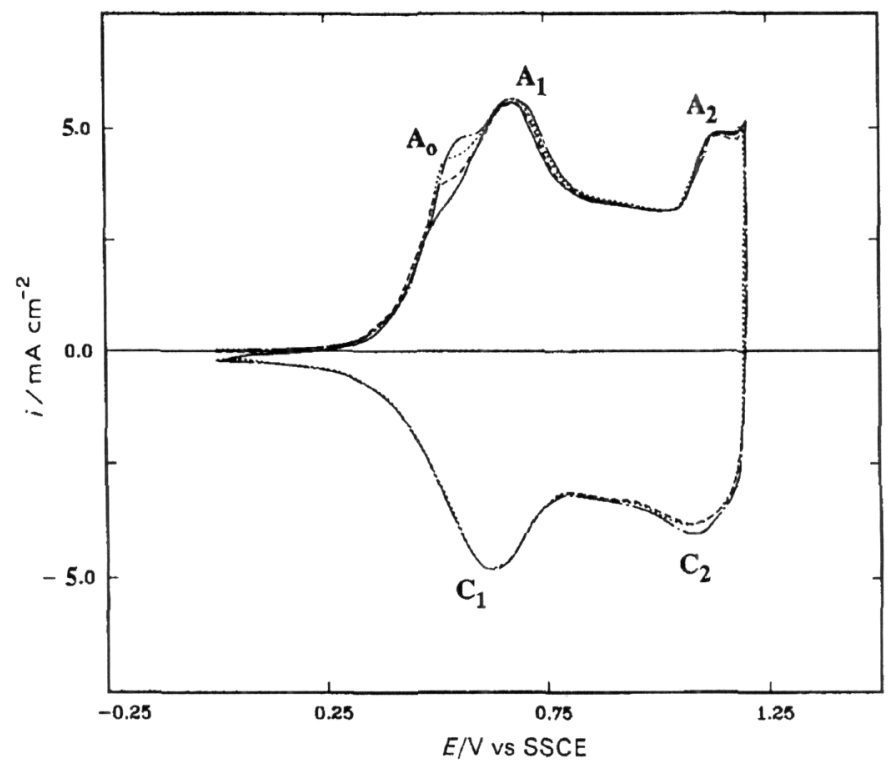

Fig. 3. Cyclic voltammetric response, $100 \mathrm{mV} \mathrm{s}^{-1}, 0.5 \mathrm{M} \mathrm{H}_{2} \mathrm{SO}_{4}$, of irridium oxide film $(\mathrm{CEF}=150)$ in first potential cycle after holding at $E$. for $10 \mathrm{~s}(-), 1 \mathrm{~min}(---), 3 \mathrm{~min}(\cdots)$ ) and $7 \mathrm{~min}(-\cdot-)$. Iridium oxide was equilibrated with continuous potential cycling between holding periods at $E_{-}$.

thicknesses as a function of $\log N$, where $N$ is the number of cycles at $100 \mathrm{mV} \mathrm{s}^{-1}$ applied after holding at $-0.24 \mathrm{~V}$ for $5 \mathrm{~min}$ after oxide growth. For very thin films, no changes whatsoever are observed in the CV response with $N$. For films of medium thickness, i.e. a CEF of about 150, little change is seen in peaks $\mathrm{A}_{1} / \mathrm{C}_{1}$.

It is important to stress the fact that these variations in the iridium oxide electrochemical response make it difficult to obtain precise kinetic data for this system in these solutions. Figure 3 shows the effect of various lengths of time spent at $E_{-}$during potential step experiments on the subsequent anodic response. Even for films of less than medium thickness, it can be seen that longer holding times lead to positive shifts of peak $A_{0}$, in particular.

Film ageing effects are also seen if $\mathrm{CV}$ runs at standard sweep rates are interspersed with very slow sweep runs. The subsequent $\mathrm{CV}$ at the original sweep rate then shows an aged $\mathrm{CV}$ response, with smaller charges being passed in the cathodic sweep, indicative of diminished reaction kinetics (the full charge can be recovered at slower sweep rates, showing that the oxide film is not lost from the surface). It is noteworthy that irridium oxide unageing can be achieved rapidly if the potential is held for some time at $E_{+}$. Therefore, as time at $E_{-}$can rapidly age the film, while time at $E_{+}$unages it, the adjective

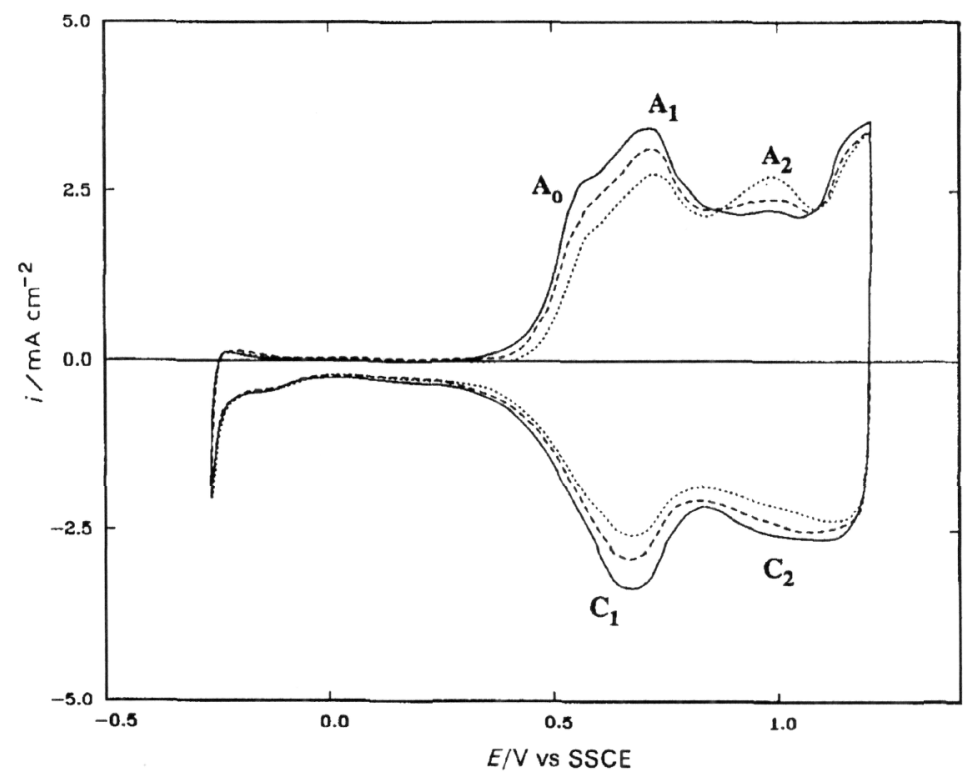

Fig. 4. Cyclic voltammograms at $100 \mathrm{mV} \mathrm{s}^{-1}$ in $1 \mathrm{M} \mathrm{HNO}_{3}$ of iridium oxide $(\mathrm{CEF}=164)$ in the first $(-\ldots), 10$ th $(\cdots--)$ and 20 th $(\cdots)$ cycle of potential after oxide growth and potential holding at $1.2 \mathrm{~V}$ for $5 \mathrm{~min}$. 

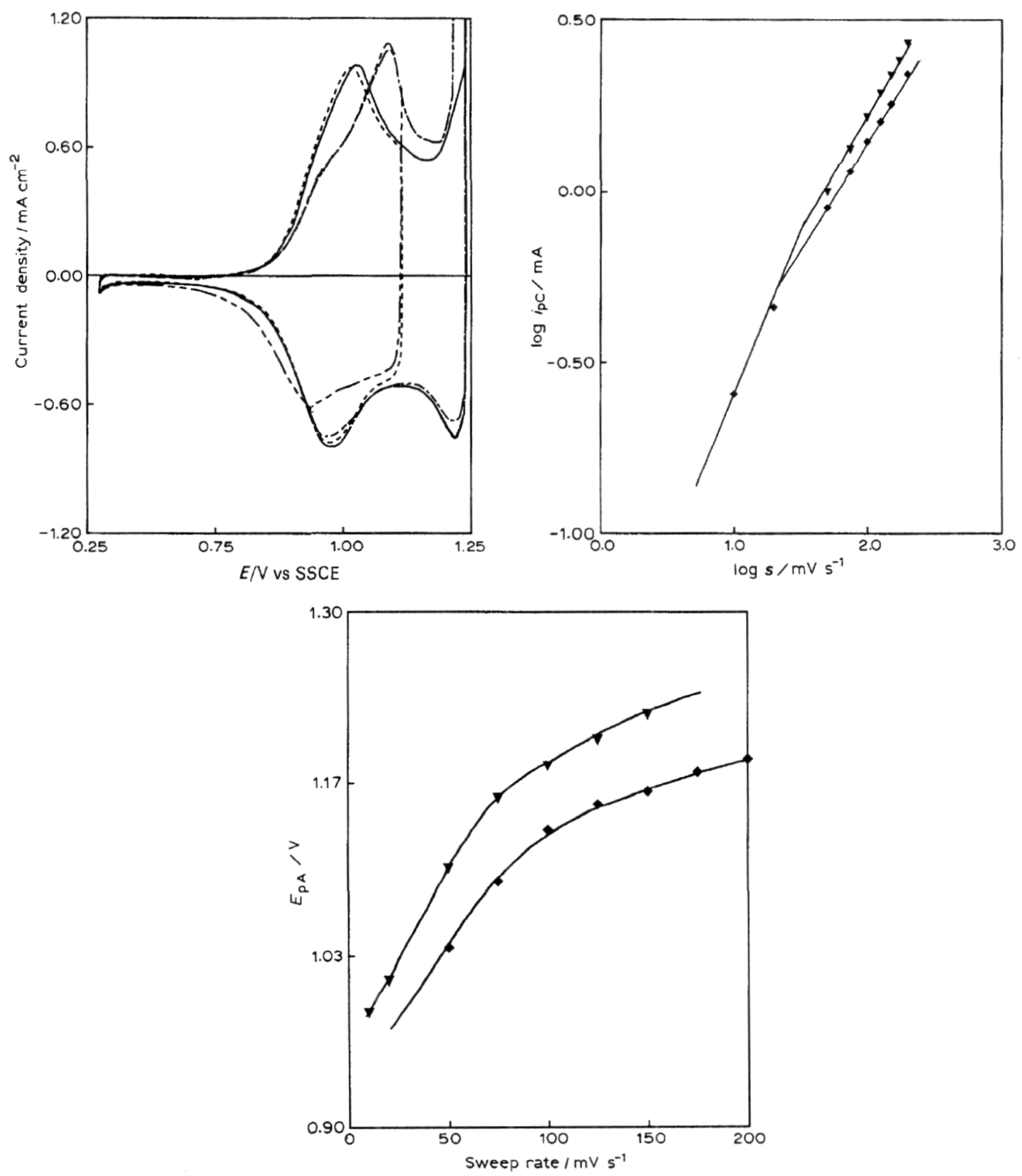

Fig. 5. (a) CV response of fresh iridium oxide (solid line) at $100 \mathrm{mV} \mathrm{s}^{-1}$, immediately after slow sweep experiments $(---$ and ----$)$ and after oxide unageing by holding at $1.25 \mathrm{~V}$ for one minute $(---)$. (b) Dependence of peak $C_{1}$ on sweep rate for aged $(\downarrow)$ and unaged $(\boldsymbol{\nabla})$ oxide film. (c) Dependence of potential of peak $A_{1}$ on sweep rate for aged ( $\left.\mathbf{v}\right)$ and unaged ( $\bullet$ ) oxide film.

'reversible' accurately describes this form of iridium oxide ageing.

In $1 \mathrm{M}$ nitric acid solutions, a similar phenomenon to that described above can be seen. However, a very different behaviour can be seen in this medium, and also in sulphuric and nitric acid solutions, which are less than $\sim 0.2 \mathrm{M}$. In these cases, the principal $\mathrm{CV}$ peaks $\left(A_{1} / C_{1}\right)$ decrease in magnitude, while the second anodic peak, $A_{2}$, increases in size with subsequent potential cycling (Fig. 4). This phenomenon is not reversible in nature and is also associated with diminished reaction kinetics. This irreversible form of ageing, also reported earlier for iridium oxide in $\mathrm{HClO}_{4} / \mathrm{LiClO}_{4}$ solutions [18], is examined in more detail in another paper currently in preparation [34].

\subsection{Kinetic limitations of reversibly aged iridium oxide films}

The rate of oxidation/reduction of iridium oxide films has been explored in a number of previous studies $[6,10-12,15,16,18,33]$. According to Conway et al. $[7,8]$, irridium oxide can be viewed as an adsorbed thin layer and the $\operatorname{Ir}(\mathrm{III}) /(\mathrm{IV})$ reaction kinetics can be 
established from the $s_{0}$ parameter (i.e. the sweep rate at which the relationship between peak current and sweep rate changes from linear to square root) obtained from CV experiments. Further studies by Burke et al. [10-12] and Pickup and Birss [18] have indicated that site-to-site electron hopping may be rate limiting and have resulted in a model for electrochemically formed iridium oxide which is more polymeric in nature. These latter studies have frequently shown unusual positive shifts of the anodic peaks at high sweep rates.

In the present work, the impact of the reversible ageing of iridium oxide on its reduction/oxidation kinetics is shown. Figure 5(a) (solid) shows a typical $\mathrm{CV}$ obtained for an equilibrated freshly-formed iridium oxide film. Following this, a series of CVs were recorded at slower sweep rates, down to $2 \mathrm{mV} \mathrm{s}^{-1}$. When the scan rate was increased once again to $100 \mathrm{mV} \mathrm{s}^{-1}$, peak $A_{1}$ was shifted substantially positively (see Fig. 5(a) (-- -)) and the charge passed in the cathodic scan was somewhat diminished, i.e. the response is now aged. Holding the potential at $1.25 \mathrm{~V}$ for one minute was required to unage the oxide (see Fig. 5(a) (- - )). Figure 5(b) shows the $i_{\mathrm{p}} / \log s$ dependence of peak $\mathrm{C}_{1}$, for the reversibly aged and unaged oxide films. It is seen that the apparent $s_{0}$ parameter has decreased from about $35 \mathrm{mV} \mathrm{s}^{-1}$ in the unaged condition to about $20 \mathrm{mV} \mathrm{s}^{-1}$ for the aged oxide film. This shows clearly that reversible ageing is characterized by a diminishment in iridium oxide kinetics by almost a factor of two in these experiments. Figure 5(c) indicates the substantial effect of film ageing on the potential of peak $A_{1}$, where the anodic process is shifted to more positive potentials after the slow sweep experiments.

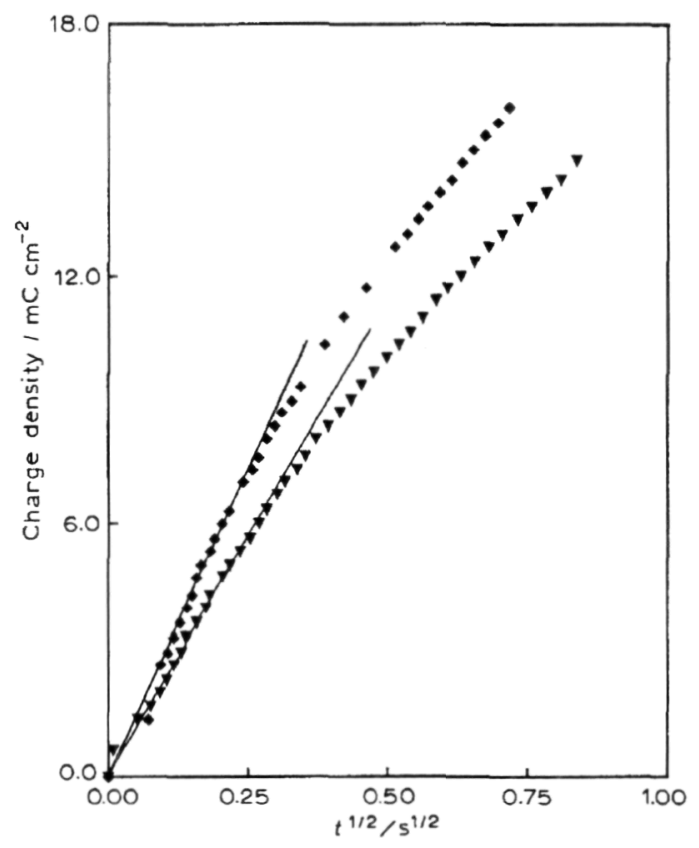

Fig. 6. $q / t^{1 / 2}$ plots for cathodic step from $1.25 \mathrm{~V}$ to $0.2 \mathrm{~V}$ for aged $(\boldsymbol{\nabla})$ and unaged $(\bullet)$ oxide film.
Previous kinetic studies with electrochemically formed iridium oxide involving potential step techniques [18] have shown that the charge passed is linear with $t^{1 / 2}$ at short times, indicative of a diffusion controlled process. In the present work, the impact of reversible ageing on the chronocoulometric data is also examined. Figure 6 shows the cathodic $q / t^{1 / 2}$ plot obtained for a freshly formed iridium oxide film of $\mathrm{CEF}=140$ for a potential step from 1.25 to $0.2 \mathrm{~V}$, from which a $D^{1 / 2} C$ parameter [18] of $2.7 \times 10^{-7} \mathrm{~mol} \mathrm{~cm}^{-2} \mathrm{~s}^{-1 / 2}$ could be obtained. When this experiment was repeated immediately after a $5 \mathrm{mV} \mathrm{s}^{-1}$ potential scan, the $D^{1 / 2} C$ value had decreased to $2.0 \times 10^{-7} \mathrm{~mol} \mathrm{~cm}^{-2} \mathrm{~s}^{-1 / 2}$. Similar to the results of Fig. 5(b), this also serves to demonstrate that reversible iridium oxide ageing is characterized by the deterioration of its kinctics. This can also be seen readily by examining the effect of various times spent in the reduced state of iridium oxide on the $D^{1 / 2} C$ values obtained from subsequent anodic transients.

These results show clearly that the electrochemical response of iridium oxide, and in particular, the associated kinetics of film oxidation/reduction, are highly variable in these solutions. Clearly, the measured kinetics depend on oxide film thickness, time spent in a particular range of potential, prior sweep rates used, and so on. Most importantly, aged films display substantially poorer kinetics than do unaged films. The connection between reversibly aged films and slow kinetics can be seen even more clearly by comparing the aged $\mathrm{CV}$ response in the first cycle of potential in Fig. 1 with the $\mathrm{CV}$ response for an unaged film $(\mathrm{CEF}=332)$ at high sweep rates (Fig. 7), as well as the unaged response in Fig. 1 with that obtained for the oxide film in Fig. 7 at a rather slow sweep rate.

\subsection{Iridium oxide behaviour in basic solutions}

A positive shift of the principal anodic peak in the case of polyaniline films and iridium oxide and the appearance of pre-peaks such as $\mathrm{A}_{0}$ for sputtered iridium oxide electrodes have recently been attributed [30] to resistance effects in these films. Although this latter study did not specifically refer to shifts of the $A_{1}$ and $A_{0}$ peaks of iridium oxide as a function of experimental variables such as film thickness and time spent in the reduced state, it did relate these effects to a change in film resistance, which is suggested to occur as the film is converted from the non-conducting $\operatorname{Ir}(\mathrm{III})$ form to the conducting $\operatorname{Ir}(\mathrm{IV})$ form. This interpretation of the origin of prepeaks and peak shifts would imply that the kinetics of film oxidation would be slow initially, but should then accelerate as oxidation proceeds, i.e. as the ratio of the number of $\operatorname{Ir}(\mathrm{IV})$ to $\operatorname{Ir}(\mathrm{III})$ sites increases.

If this is the correct interpretation of the reversible ageing effects described here for iridium oxide, then this form of ageing should also be observed in basic solutions. For this reason, iridium oxide films were 


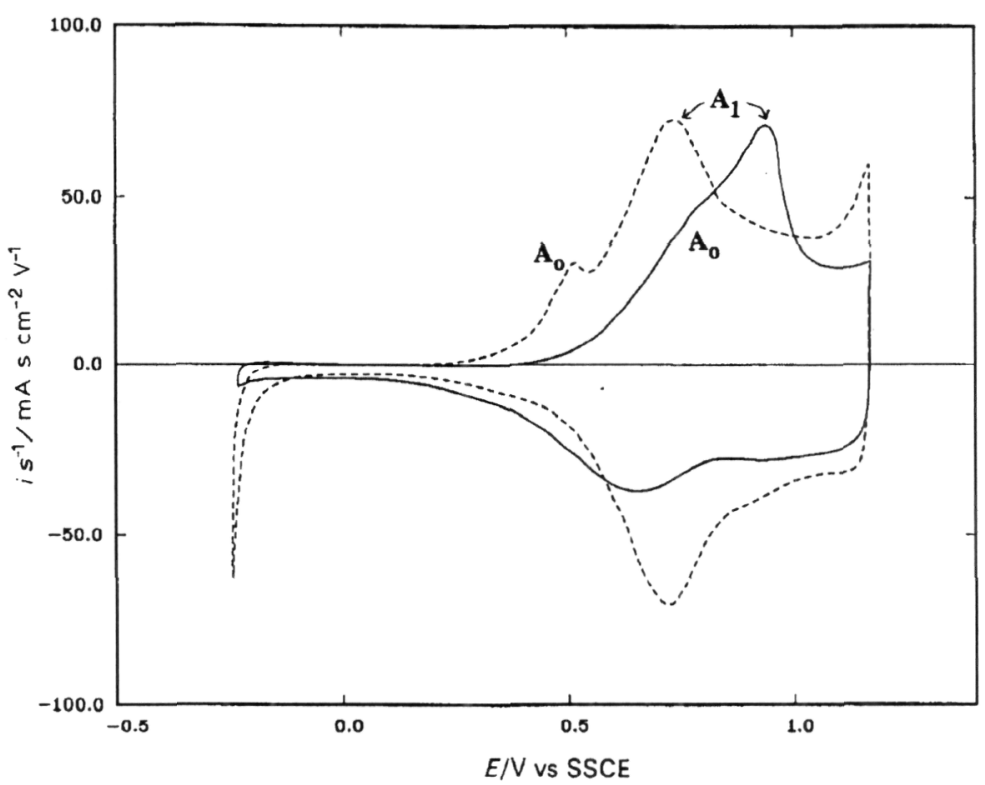

Fig. 7. Cyclic voltammograms of iridium oxide $(\mathrm{CEF}=332)$ in $0.5 \mathrm{M} \mathrm{H}_{2} \mathrm{SO}_{4}$ at $200 \mathrm{mV} \mathrm{s}^{-1}(-)$ and $5 \mathrm{mV} \mathrm{s}^{-1}(-\cdots)$.

also carcfully examined in alkaline media in the present work. The growth of iridium oxide films is known to be difficult in basic solutions [12], and therefore, oxides were grown in acidic solutions and then transferred to $\mathrm{NaOH}$ solutions. The effect of time at $E_{-}$and $E_{+}$in the acidic growth solution immediately prior to transfer of the oxide, as well as the impact of time at constant potentials in alkaline solutions, was then investigated.

In the first set of experiments, a relatively thick iridium oxide film $(\mathrm{CEF}=298)$ was formed in sulphuric acid, and the characteristic CV response (Fig. 8 , curve (a)) for such a film was obtained. The potential was then maintained at $E_{-}$for some time, where, by inspection of Reaction 1, it can be seen that a fully reduced iridium oxide films should contain a substantial amount of $\mathrm{H}^{+}$and $\mathrm{SO}_{4}^{2-}$ when in the sulphuric acid solution.

Upon transfer of the iridium oxide at $E_{-}$into a $1 \mathrm{M}$ $\mathrm{NaOH}$ solution, (Reaction 2 indicates that the oxide films would be expected to be free of $\mathrm{Na}^{+}$and $\mathrm{OH}^{-}$ ions under this condition and that only water should be present), the first anodic scan (Fig. 8, curve (b)) reveals a response which, by analogy to acidic solutions, would be termed as very aged. However, within only one or two complete cycles of potential in the basic solution, these shifts are completely erased (Fig. 8, curves (c)), contrary to the case in the sulphuric acid medium, where long cycling times would have been required. It is possible that the shift observed in the first scan in base reflects the fact that the oxide film still contains a significant amount of sulphuric acid, and hence behaves as if it were being studied in this solution. During the anodic sweep, sulphate ions and protons would then be expelled from the oxide, as usual.

It is of interest to note that when the film is transferred back to sulphuric acid, an unaged response is seen (Fig. 8, curve (d)). Also, if a thin iridium oxide film is transferred at $E_{\text {- }}$ from sulphuric acid to basic solutions, no shifts in the peaks are observed in the first or subsequent cycles.

A further probe of the similarities and differences of the ageing characteristics of iridium oxide in acidic and basic media was made by investigating the effect of time spent at $E_{-}$in basic solutions after oxide equilibrium in base was complete. The only effect of time at $E_{-}$was to accomplish the complete reduction of the film (as the sweep rate in the prior cathodic scan was too rapid to permit the complete reaction of a film of this thickness), i.e. no ageing was seen.

It is also interesting that when a relatively thick oxide of a similar charge density as in Fig. 8 is removed from the sulphuric acid medium at $E_{+}$such that the solution ions (sulphate and protons) would be expected to have been expelled from the oxide film prior to its transfer, the first and subsequent scans in base show no shifts or anomalies which are characteristic of an aged response. This can be interpreted as reflecting a greater ease of equilibration of the contents of the oxide film when an essentially ion-free oxide film is transferred from acidic to basic solutions.

\subsection{Possible origins of reversible ageing}

The focus of this paper has been on elucidating the factors which lead to the reversible ageing/unageing of the response of electrochemically formed iridium oxide films, particularly in acidic solutions. The most important parameter appears to be the time spent in the reduced state, particularly for thick films. Under these conditions, diminished reaction kinetics are observed and a brief period of time at positive potentials or long times of continuous cycling are required to reverse the effect, i.e. to unage the films. 


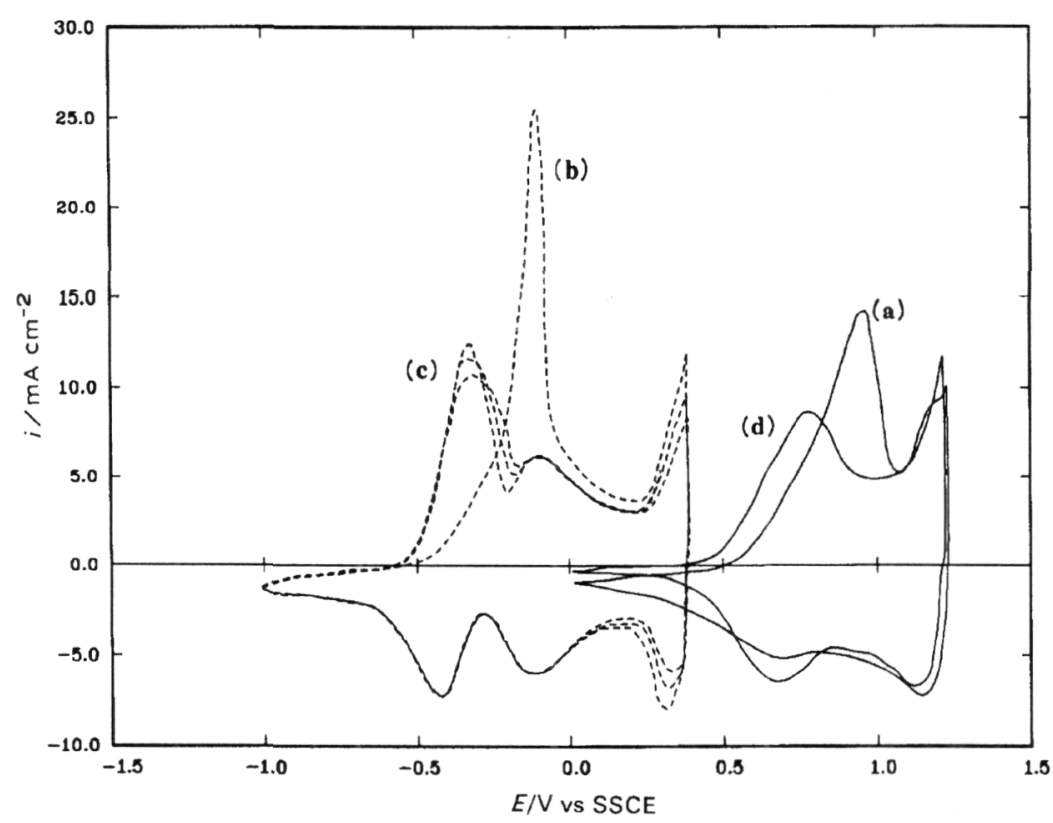

Fig. 8. Cyclic voltammetric response of a thick iridium oxide film ( $\mathrm{CEF}=298), 100 \mathrm{mV} \mathrm{s}^{-1}$ : (a) after growth and cycling in $0.5 \mathrm{M} \mathrm{H}_{2} \mathrm{SO}_{4}$; (b) first cycle after transfer at $E_{-}$to $1 \mathrm{M} \mathrm{NaOH}$; (c) after equilibration in base; (d) after transfer back to $0.5 \mathrm{M} \mathrm{H}_{2} \mathrm{SO}_{4}$.

It is possible that the process occurring in peak $A_{0}$ may have an important bearing on these film ageing phenomena. It has been reported previously [7-9] that peak $\mathrm{A}_{0}$ is more pronounced when iridium oxide films are grown in carbonate-containing solutions, than in sulphuric acid media, and it was claimed that the $A_{0}$ peak may be related to the presence of anions, e.g. carbonate, in the oxide film. It has also been shown $[7,8]$ that both the reaction occurring in peak $A_{0}$ and in its cathodic counterpart are kinetically slow. Time at negative potentials would then result in the complete reduction of the $A_{0}$ sites, which would then result in its slow oxidation, seen as a positive shift in the anodic currents in the next cycle. However, this does not explain why many cycles of potential are required to fully unage the oxide film (Fig. 1). Also, it does not clarify why oxide reduction kinetics, in particular, are inhibited after film ageing.

Reversible ageing of iridium oxide films in acidic solutions are more likely to be related specifically to the ion/solvent injection/expulsion processes which have been suggested to occur during film oxidation/ reduction (Reaction 1). In the following discussion, the similarities of the indicators of reversible ageing of iridium oxide films to those observed at other electrode systems, particularly for a number of polymer electrodes, and the proposed origins of this effect in these cases will be discussed. For example, after holding the potential of a PCMS-VC 16 -coated gold electrode at negative values for some time [29], the anodic peak shifts positively in the next anodic scan. With continuous potential cycling of the potential, the peak gradually moves negatively to its steadystate position. These results were explained in terms of the shrinking/swelling of the polymer film which accompanies the film reduction/oxidation processes, respectively. Swelling of the polymer film was sug- gested to occur during the anodic cycle due to the injection of ions and associated solvent into the film. Polymer shrinking, a slower process than swelling, appeared to require time at negative potentials, resulting in the gradual loss of ions/solvent from the reduced form of the film.

The origin of the swelling during oxidation was linked to the higher positive charge of the oxidized form of these types of films as compared to the reduced. This leads to the need for anion and solvent injection during oxidation, and vice versa during reduction. Without potential holding at $E_{-}$, insufficient time is available for all ions and solvent to be expelled from the film. Therefore, the ion/solvent injection process in the next oxidation step is facilitated and the anodic CV peak appears kinetically more reversible and is symmetrical to the cathodic one. With time spent at $E_{-}$, the complete loss of ions and solvent from the film causes film shrinkage, thus inhibiting the next ion/solvent injection step during oxidation, leading to a positively shifted anodic peak.

The reversible ageing of iridium oxide, particularly in sulphuric acid solutions, closely resembles these polymer systems, not only phenomenologically, but also in terms of the involvement of ions/solvents in the oxide reaction (Reactions 1 and 2). Recent results obtained using the QCMB technique [24] have shown that, during iridium oxide reduction in sulphuric acid solutions, sulphate ions and protons are injected into the film, while water is expelled. Therefore, the common thread between these results and the polymer electrode behaviour is the loss of solvent from the film at negative potentials. Therefore, as a result of long times spent in the reduced state, particularly for thick irridium oxide films, it is suggested that some film dehydration occurs, leading to a partial 
loss of the advantageous hydrous properties of these materials. This loss of solvent leads to slower reaction kinetics (Figs 5 and 6), while time spent in the oxidized form of the film accelerates the recovery process. The QCMB data indicates [24] that water is regained into the oxide film under these latter conditions.

The QCMB data obtained in alkaline solutions has indicated [24] that at negative potentials, while $\mathrm{Na}^{+}$ and $\mathrm{OH}^{-}$ions are expelled from the film, water is injected. This increase in the water content of the film in the reduced state would be consistent with the observed absence of film ageing in these solutions. The lack of ageing effects in basic solutions also supports the notion that ageing is not simply the result of the complete reduction of the Ir(IV) sites within the oxide film, leading to a non-conducting $\operatorname{Ir}(\mathrm{III})$ oxide film. Rather, film ageing is related to the ion/solvent content of these films, which is very different at negative potentials in acidic vs alkaline solutions.

\section{Summary}

The cyclic voltammetric response of iridium oxide films, grown electrochemically in sulphuric acid solutions, can exhibit a notable dependence on time spent in the reduced state, similar to what has been reported previously for a number of polymer-coated electrode systems. This effect is seen primarily by a pronounced positive shift of the principal anodic peak, and a decrease in the charge passed in the cathodic sweep. It reflects a diminishment in reaction kinetics by at least a factor of two and is therefore described as film ageing. The effect is more significant the thicker the oxide film. The films can be unaged either by long periods of time of continuous cycling or by a brief time at relatively positive potentials. It is therefore described as a reversible ageing phenomenon.

As film ageing is induced primarily by time spent in the reduced state in acidic solutions and as films can be unaged rapidly at positive potentials, it is suggested that film ageing is associated with partial dehydration of the oxide film, slowing down the kinetics of oxidation/reduction until film hydration is reaccomplished. This is supported by recent results obtained with the QCMB technique [24], which indicated that, in acidic solutions, ion injection during film reduction is accompanied by loss of water, and vice versa during film oxidation. This is also consistent with the absence of ageing effects in alkaline media, where time at negative potentials was shown by QCMB experiments to bring about ion expulsion and water injection.

\section{Acknowledgements}

The authors wish to express their appreciation to the Natural Sciences and Engineering Research Council of Canada for the support of this work and to Jeff Segal for technical assistance.

\section{References}

[1] A. Capon and R. Parsons, J. Electroanal. Chem. 39 (1972) 275.

[2] D. N. Buckley and L. D. Burke, J. Chem. Soc. Faraday Trans. 171 (1975) 1447.

[3] J. O. Zerbino, N. R. de Tacconi and A. J. Arvia, J. Electrochem. Soc. 125 (1978) 1266.

[4] S. Gottesfeld and S. Srinivasan, J. Electroanal. Chem. 86 (1978) 89 .

[5] S. Gottesfeld and J. D. E. McIntyre, J. Electrochem. Soc. 126 (1980) 742.

[6] S. H. Glarum and J. H. Marshall, ibid. 127 (1980) 1457.

[7] J. Mozota and B. E. Conway, ibid. 128 (1981) 2142.

[8] Idem, Electrochim. Acta 28 (1983) 1, 9.

[9] V. I. Birss, R. Meyers, H. Angerstein-Kozlowska and B. E. Conway, J. Electrochem. Soc. 131 (1984) 1502.

[10] L. D. Burke, J. K. Mulcahy and D. P. Whelan, J. Electroanal. Chem. 163 (1984) 117.

[11] L. D. Burke and D. P. Whalen, ibid. 162 (1984) 121

[12] L. D. Burke and R. A. Scannell, ibid. 175 (1984) 119

[13] D. A. J. Rand and R. Woods, ibid. 55 (1984) 375

[14] P. G. Pickup and V. I. Birss, ibid. 220 (1987) 83.

[15] P. G. Pickup and V. I. Birss, J. Electrochem. Soc. 135 (1988) 126.

[16] Idem, J. Electroanal. Chem. 240 (1988) 171

[17] Idem, J. Electrochem. Soc. 135 (1988) 41.

[18] Idem, J. Electroanal. Chem. 240 (1988) 185.

[19] M. L. Hitchmann and S. Ramanathan, Analyst 113 (1988) 35.

[20] M. Vukovic, J. Appl. Electrochem. 17 (1989) 737.

[21] D. Cukman and M. Vukovic, J. Electroanal. Chem. 279 (1990) 283.

[22] M. Vukovic, J. Appl. Electrochem. 20 (1990) 969.

[23] R. Kotz, C. Barbero and O. Haas, J. Electroanal. Chem. 296 (1990) 37.

[24] V. I. Birss, H. Elzanowska and S. Gottesfeld, ibid. 318 (1991) 327.

[25] L. S. Robblee, J. L. Lefko and S. B. Brummer, J. Electrochem. Soc. 130 (1983) 731.

[26] C. L. Ballestrasse, R. T. Ruggeri and T. Beck, Ann. Biomed. Eng. 13 (1985) 405.

[27] A. H. Schroeder, F. B. Kaufman, V. Patel and E. M. Engler, J. Electroanal. Chem. 113 (1980) 193.

[28] A. H. Schroeder and F. B. Kaufman, ibid. 113 (1980) 209

[29] Yu-Min Tsou, Hsu-Yang Liu and A. J. Bard, J. Electrochem. Soc. 135 (1988) 1669

[30] S. Gottesfeld, A. Redondo, I. Rubinstein and S. W. Feldberg, J. Electroanal. Chem. 265 (1989) 15.

[31] P. Daum and R. W. Murray, J. Phys. Chem. 85 (1981) 389.

[32] H. S. White, J. Leddy and A. J. Bard, J. Amer. Chem. Soc. 104 (1982) 481.

[33] K. Lian and V. I. Birss, J. Electroanal. Chem. 319 (1991) 227.

[34] H. Elzanowska, J. Scgal and V. I. Birss, J. Electroanal. Chem., submitted.

[35] Idem, J. Electrochem. Soc., submitted.

[36] H. A. Kozlowska and B. E. Conway, J. Electroanal. Chem. 95 (1979) 273. 